\title{
Geraniol induces cooperative interaction of apoptosis and autophagy to elicit cell death in PC-3 prostate cancer cells
}

\author{
SU-HWA KIM ${ }^{1}$, EUN-JUNG PARK ${ }^{1}$, CHAE RYUN LEE ${ }^{1}$, JUNG NYEO CHUN ${ }^{1}$, NAM-HYUK CHO ${ }^{2}$, IN-GYU KIM ${ }^{3}$ \\ SANGHOON LEE ${ }^{4}$, TAE WOO KIM ${ }^{5}$, HYUN HO PARK ${ }^{6}$, INSUK SO ${ }^{1,7}$ and JU-HONG JEON ${ }^{1,7}$ \\ Departments of ${ }^{1}$ Physiology, ${ }^{2}$ Microbiology and Immunology, and ${ }^{3}$ Biochemistry and Molecular Biology, Seoul National \\ University College of Medicine, Seoul 110-799, Republic of Korea; ${ }^{4}$ Department of Biomedical Informatics, \\ University of Utah, Salt Lake City, UT 84112, USA; ${ }^{5}$ Division of Oncology, Graduate School of Medicine, \\ Korea University, Seoul 136-701; ${ }^{6}$ Department of Biotechnology, Research Institute of Protein Sensor, \\ Yeungnam University, Gyeongsan 712-749; 7 Institute of Dermatological Science, \\ Medical Research Center, Seoul National University, Seoul, Republic of Korea
}

Received October 17, 2011; Accepted December 2, 2011

DOI: 10.3892/ijo.2011.1318

\begin{abstract}
Geraniol, an acyclic dietary monoterpene, suppresses prostate cancer growth and enhances docetaxel chemosensitivity in cultured cell or xenograft tumor models. However, the mechanisms of the geraniol action against prostate cancer are largely unknown. In this study, we investigated the cellular and molecular mechanisms of geraniol-induced cell death in PC-3 prostate cancer cells. Among the examined structurally and functionally similar monoterpenes, geraniol potently induced apoptosis and autophagy. Although independent processes, apoptosis and autophagy acted as cooperative partners to elicit geraniol-induced cell death in PC-3 cells. At a molecular level, geraniol inhibited AKT signaling and activated AMPK signaling, resulting in mTOR inhibition. Combined treatment of AKT inhibitor and AMPK activator markedly suppressed cell growth compared to either treatment alone. Our findings provide insight into future investigations that are aimed at elucidating the role of apoptosis and autophagy in prostate cancer therapy and at developing anticancer strategies co-targeting AKT and AMPK.
\end{abstract}

\section{Introduction}

Geraniol is a naturally occurring acyclic monoterpene derived from herb oils (1). Previous in vivo tumor model studies show that geraniol exerted chemotherapeutic or chemosensitizing activity against several types of cancers, including colon, pancreatic, hepatic, and prostate cancer (2-5). Geraniol reduces

Correspondence to: Dr Ju-Hong Jeon, Department of Physiology, Seoul National University College of Medicine, 103 Daehak-ro, Jongno-gu, Seoul 110-799, Republic of Korea

E-mail: jhjeon2@snu.ac.kr

Key words: geraniol, apoptosis, autophagy, cell death, prostate cancer mevalonate metabolism by inhibiting HMG-CoA reductase activity (6), which may contribute to its anticancer activity. However, the cellular and molecular mechanisms of anticancer action of geraniol are poorly understood. In addition, although certain types of monoterpenes, such as menthol, linalool, and cineol, are structurally and functionally similar to geraniol (7), the specificity of therapeutic actions of geraniol are only slightly evaluated.

Prostate cancer is one of the most common malignancies and the leading causes of cancer mortality in males (8). Metastatic prostate cancers initially respond to androgen deprivation therapy, but eventually progress into castration-resistance prostate cancers that acquire the ability to evade cell death in androgen-depleted conditions and become resistant to current therapeutic regimens $(9,10)$. Therefore, strategies to restore the ability to induce cell death are required to improve therapies for prostate cancer. However, cell death is a complex biological phenomenon that is independently or interactively regulated by multiple cellular processes, including apoptosis and autophagy (11). Although apoptosis and autophagy share common molecular regulators or pathways, they exhibit synergistic or antagonistic effects on each other during cell death, depending on the cell type and/or context (12-14). The relationship between apoptosis and autophagy is largely unknown in prostate cancer.

Aberrant activation of AKT signaling mediates the inhibition of apoptosis and autophagy $(15,16)$, which may contribute to cancer progression and chemoresistance. In prostate cancer cells, AKT enhances cell survival and increases resistance to cell death under androgen-depleted conditions via various mechanisms, including mTOR activation (17). PTEN, a tumor suppressor gene, antagonizes PI3K activity by dephosphorylating PIP3, which inhibits AKT activity (18). Loss of PTEN has been observed in approximately $70 \%$ of metastatic prostate cancer samples $(19,20)$, which suggests that PTEN-mediated inhibition of AKT is frequently attenuated in metastatic prostate cancer. In addition, androgen receptor inhibition activates AKT by reducing the expression level of PHLPP, an AKT phosphatase (21). AMPK also interferes with AKT signaling by phosphory- 
lating TSC2 and/or Raptor, which inhibits mTOR activity (22). AMPK inhibition accelerated cell proliferation and promoted malignant behavior (23). These findings suggest that the anticancer effects of AKT inhibition may be potentiated by AMPK activation.

In the xenograft tumor model using $\mathrm{PC}-3$ prostate cancer cells, geraniol suppressed prostate cancer growth and enhanced docetaxel chemosensitivity (5). In this study, we investigated the mechanisms of geraniol action against prostate cancer. We found that geraniol induces apoptosis and autophagy, which function as cooperative partners to elicit cell death in PC-3 cells. We also showed that geraniol inhibits AKT activity and activates AMPK activity. Combined treatment of AKT inhibitor and AMPK activator markedly suppressed cell growth compared to either treatment alone. Our findings provide insight into future investigations that are aimed at elucidating the role of apoptosis and autophagy in prostate cancer therapy and at developing the anticancer strategies co-targeting AKT and AMPK. In addition, further studies using geraniol may help understand the phenotypic complexity of the relationship between apoptosis and autophagy at the molecular level.

\section{Materials and methods}

Cell culture and reagents. The PC-3 cells were obtained from ATCC and maintained in DMEM containing 10\% fetal bovine serum. All cell culture agents were purchased from Invitrogen. Geraniol, (-)-menthol, linalool, cineol, 3-methladenine (3-MA), mevalonate, and okadaic acid were purchased from Sigma. Tautomycin and zVAD-fmk (zVAD) were obtained from Calbiochem. Geraniol, (-)-menthol, linalool, and cineol were dissolved in ethanol ( $0.1 \%$ working concentration) as a vehicle.

Cell viability assay. PC-3 cells were grown in 12-well culture plates (Nunc). MTT and LDH release assays were used to assess cell viability according to the manufacturer's instructions (Sigma-Aldrich and Promega, respectively). The assays were quantitated by measuring the absorbance at 570 (MTT assay) or $490 \mathrm{~nm}$ (LDH assay) using a microplate spectrophotometer (Biochrom).

Caspase-3 activity assay. Capase-3 activity was determined using a commercial kit according to the manufacturer's instruction (Biomol). The crude extracts from PC-3 cells were incubated with chromogenic caspase- 3 substrates. The absorbance was measured at $405 \mathrm{~nm}$ using a microplate spectrophotometer.

Flow cytometric analysis. To analyze cell cycle profiles, PC-3 cells were fixed with $70 \%$ ethanol and labeled with propidium iodide $(50 \mu \mathrm{g} / \mathrm{ml})$ solution containing RNase A $(100 \mu \mathrm{g} / \mathrm{ml})$. The cells were analyzed using flow cytometry (BD Biosciences). ModFitLT V3.0 software was used for data analysis.

GFP-LC3 puncta analysis. PC-3 cells were seeded on glass coverslips and transfected with GFP-LC3 plasmid using Lipofectamine 2000 (Invitrogen). The cells expressing GFP-LC3 were directly observed using the FluoView 1000 confocal microscope (Olympus). The number of GFP-LC3 puncta in each cell (total 40-50 cells) was counted in at least five independent visual fields.
Western blot analysis. The crude extracts of PC-3 cells were prepared by incubation with RIPA buffer containing protease and phosphatase inhibitor cocktails (Calbiochem). The extracts were resolved using 6\%-15\% SDS-PAGE gels and probed with the indicated antibodies. Antibodies against ATG5, cleaved caspase-3, Bcl-xL, pAKT, AKT, pGSK-3 $\beta$, pmTOR, mTOR, pS6K, S6K, p4EBP, 4EBP, pACC, ACC, pAMPK, pAMPK, pPDK1, PDK1, pERK, ERK, pp38, p38, and pJNK were obtained from Cell Signaling Technology. The anti-LC3 antibody was purchased from MBL. Antibodies against Bcl-2, Bax, GSK- $3 \beta$, JNK, and GAPDH were purchased from Santa Cruz Biotechnology. The data are representative of at least three independent experiments.

Electrophysiology. Patch clamp experiments were performed in the whole cell configuration using an Axopatch I-D amplifier according to our previous study (24). The current-voltage relationship was evaluated using linear 400-ms voltage ramps from -100 to $+100 \mathrm{mV}$ at a holding potential of $-60 \mathrm{mV}$.

Intracellular $\mathrm{Ca}^{2+}$ measurement. The cytoplasmic calcium concentration $\left[\left(\mathrm{Ca}^{2+}\right) \mathrm{cyt}\right]$ was determined as previously described (25). Fluorescence emission at $510 \mathrm{~nm}$ was measured at an excitation at $340 / 380 \mathrm{~nm}$ using a fluorescence spectrophotometer (Photon Technology Instrument) in a quartz microcuvette with stirring. Maximum and minimum fluorescence values at $380 \mathrm{~nm}\left(\mathrm{~F}_{\max }\right.$ and $\left.\mathrm{F}_{\min }\right)$ were calibrated by addition of $0.2 \%$ Triton X-100 and 10 mM EGTA, respectively. The $\left[\mathrm{Ca}^{2+}\right]$ cyt was calculated using the following equation, $\left[\mathrm{Ca}^{2+}\right]=K \mathrm{~d} \times \beta \times\left(\mathrm{R}-\mathrm{R}_{\min }\right) /\left(\mathrm{R}_{\max }-\mathrm{R}\right)$ where $K$ d is the dissociation constant for Fura-2 (224 nM), $\beta$ is $F_{\text {min }} / F_{\text {max }}$, and R is F340/ F380.

Statistical analysis. The data were expressed as the mean \pm SEM. The comparison of the means among experimental groups was performed using ANOVA followed by a post hoc test. $\mathrm{P}<0.05$ was considered statistically significant.

\section{Results}

Geraniol induces apoptosis in PC-3 cells. We previously reported that geraniol induces apoptosis using in vitro cultured cells and in vivo xenograft models (5). Because certain types of monoterpenes, such as menthol, linalool, and cineol, are structurally and functionally similar to geraniol (7), we compared the anticancer effects of these monoterpenes using MTT assays in PC-3 cells. As presented in Fig. 1A, geraniol significantly suppressed the growth of PC-3 cells compared to other monoterpenes. In addition, the cell cycle profile analysis showed that geraniol potently elevated the percentage of sub-G1 phase cells compared to other monoterpenes (Fig. 1B). Geraniol-specific induction of apoptosis was confirmed using caspase-3 activity assays. Compared to other monoterpenes, geraniol strongly increased caspase-3 activity (Fig. 1C). We also confirmed that geraniol potently produces cleaved caspase- 3 , an enzymologically active form (Fig. 1D).

Because multiple cellular processes are involved in cell death $(11,12)$, we investigated whether apoptosis is the sole cellular mechanism of geraniol-induced cell death. MTT assays showed that a pan-caspase inhibitor zVAD only partially rescued 

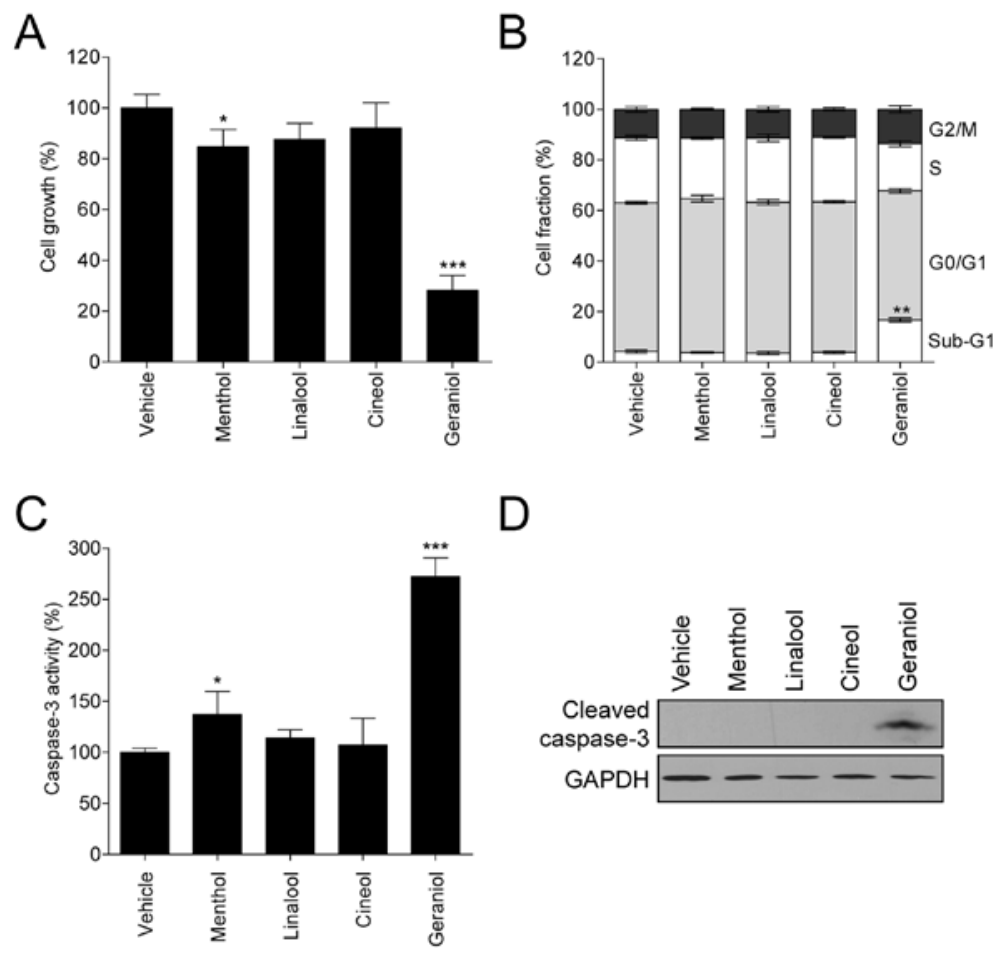

D

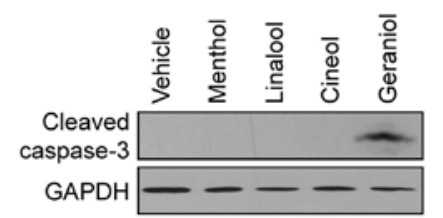

Figure 1. Geraniol induces apoptosis in PC-3 cells. (A) The cells were treated with the indicated monoterpenes (1 mM each) for $72 \mathrm{~h}$ prior to MTT assays. Cell growth was expressed as a relative absorbance value compared to that of vehicle-treated cells which was set to $100 \%$. (B) The cells were treated with the monterpenes $(1 \mathrm{mM}$ each) for $20 \mathrm{~h}$ prior to cell cycle analyses. The cell fraction was expressed as the percentage of each phase of the cell cycle. (C) The cells were incubated with the monoterpenes ( $1 \mathrm{mM}$ each) for $48 \mathrm{~h}$ prior to caspase- 3 activity assays. Caspase- 3 activity in vehicle-treated cells was expressed as $100 \%$. (D) The cells were incubated with the monoterpenes $(1 \mathrm{mM}$ each) $60 \mathrm{~h}$ prior to Western blot analyses. Equal loading was ascertained based on GAPDH levels. The data are expressed as the mean $\pm \operatorname{SEM}(\mathrm{n}=4) .{ }^{*} \mathrm{P}<0.05,{ }^{* *} \mathrm{P}<0.01,{ }^{* * *} \mathrm{P}<0.005$.

A

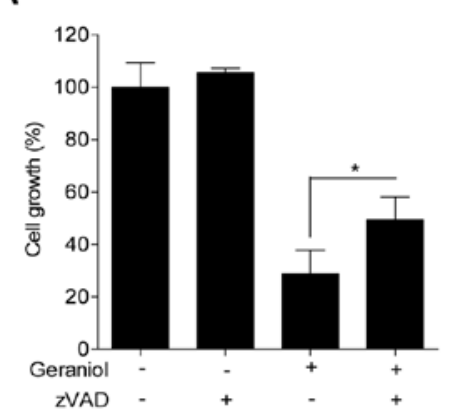

$\mathrm{D}$

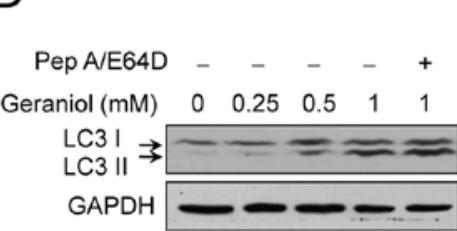

Geraniol (mM) $\quad \begin{array}{llll}0 & 0.25 & 0.5 & 1\end{array}$

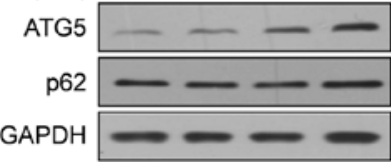

B

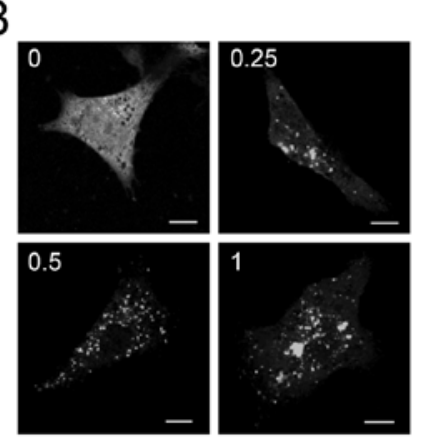

$\mathrm{E}$

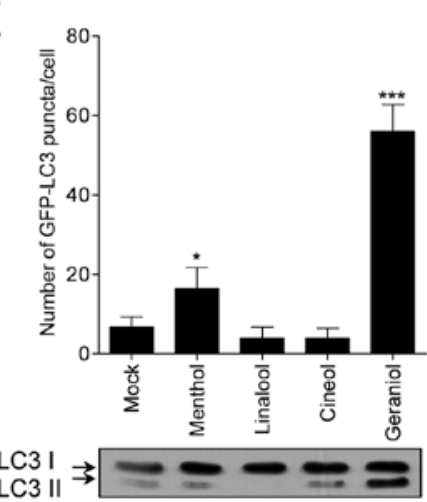

C

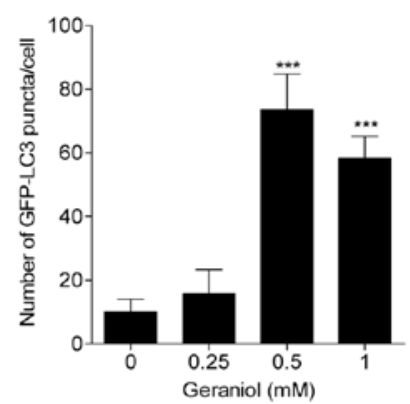

$\mathrm{F}$

Geraniol (h) $\quad 0 \quad 12 \quad 24 \quad 48 \quad 60$

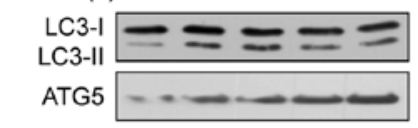

Cleaved

caspase 3 an

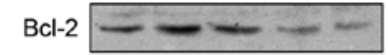

$\mathrm{Bcl}-\mathrm{xL}=-\infty-$

Bax

GAPDH

Figure 2. Geraniol induces autophagy in PC-3 cells. (A) The cells were treated with geraniol $(1 \mathrm{mM})$ and/or zVAD $(10 \mu \mathrm{M})$ for $72 \mathrm{~h}$ prior to MTT assays. The data are expressed as the mean \pm SEM $(n=4)$. ${ }^{*} \mathrm{P}<0.05$. (B and C) The cells expressing GFP-LC 3 were treated with geraniol at the indicated concentrations for $20 \mathrm{~h}$ prior to confocal microscopic analyses. The number of GFP-LC3 dots in each cell was counted in at least three independent visual fields. The data are expressed as the mean $\pm \operatorname{SEM}(n=20){ }^{* * *} \mathrm{P}<0.005$. (D) The cells were treated with geraniol at the indicated concentrations for $20 \mathrm{~h}$ and LC3-II level was determined using Western blot analysis. (E) The cells expressing GFP-LC3 were incubated with the monoterpenes ( $1 \mathrm{mM}$ each) for $20 \mathrm{~h}$ prior to counting the number of GFP-LC 3 puncta. The data are expressed as the mean \pm SEM $(n=20)$. ${ }^{*} \mathrm{P}<0.05,{ }^{* * *} \mathrm{P}<0.005$. (F) The cells were treated with geraniol at $1 \mathrm{mM}$ for the indicated times prior to Western blot analyses. 
A

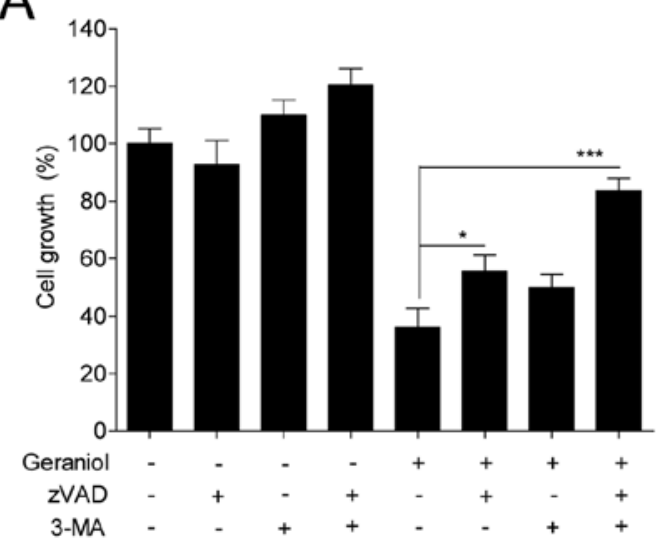

B

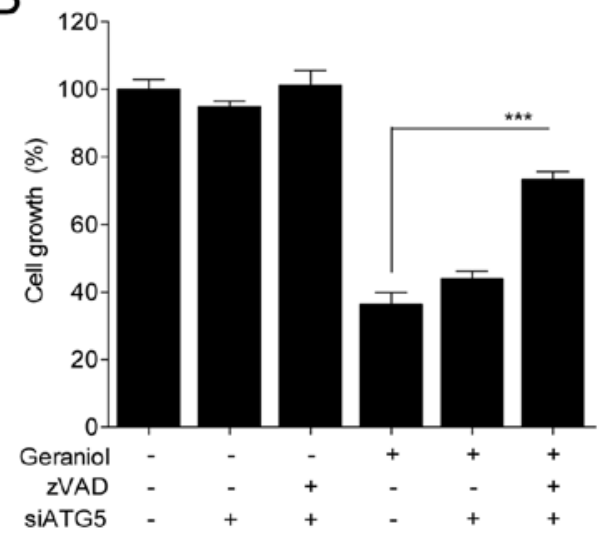

Figure 3. Combined inhibition of apoptosis and autophagy restores cell growth in PC-3 cells. (A) The cells were treated with geraniol (1 mM), zVAD (10 $\mu \mathrm{M})$, and/ or 3-MA $(2 \mathrm{mM})$ for $72 \mathrm{~h}$ prior to MTT assays. Cell growth was expressed as a relative value compared to that of the untreated cells which was set to $100 \%$. The data are expressed as the mean \pm SEM $(\mathrm{n}=4)$. (B) The cells were treated with geraniol $(1 \mathrm{mM}), \mathrm{zVAD}(10 \mu \mathrm{M})$, and/or siATG5 (50 nM) for $72 \mathrm{~h}$ prior to MTT assays. The data are expressed as the $\pm \operatorname{SEM}(\mathrm{n}=4)$.

geraniol-induced cell death (Fig. 2A). These results suggest that geraniol induces other form(s) of cell death in addition to apoptosis.

Geraniol induces autophagy in PC-3 cells. Based on accumulating, albeit controversial, evidence that autophagy elicits cell death (26,27), we determined whether geraniol induced cell death via autophagy in addition to apoptosis in PC-3 cells. We first examined the ability of geraniol to induce autophagy by quantifying the number of distinct puncta of GFP-LC3, which is an autophagy marker. When GFP-LC3 puncta were counted in each cell, the number of puncta markedly increased in geraniol-treated cells (Fig. 2B and C). Western blot analysis showed that geraniol increased the level of LC3-II, which is a biomarker of autophagy (Fig. 2D). Pepstatin A and E64D, which are lyosomal protease inhibitors, further increased the level of LC3-II (Fig. 2D). We also observed that increased expression levels of ATG5, which is a key protein of autophagy, correlated with increasing concentrations of geraniol (Fig. 2D). We examined the specificity of geraniol action on autophagy in PC-3 cells. Among the monoterpenes, geraniol potently increased the number of GFP-LC3 puncta and the level of LC-3 II (Fig. 2E). Although menthol slightly increased the number of GFP-LC3 puncta, it did not affect the level of LC-3 II. These results demonstrate that geraniol specifically induces autophagy in PC-3 cells.

To examine which process was activated first, we performed a time-course analysis of autophagy and apoptosis in geranioltreated PC-3 cells. LC-3 II and ATG5 levels were elevated following treatment with geraniol for $12 \mathrm{~h}$, and were reduced after $48 \mathrm{~h}$ (Fig. 2F). Alterations in the levels of proapoptotic (cleaved caspase-3 and Bax) and antiapoptotic proteins (Bcl$\mathrm{xL}$ ) were observed after $48 \mathrm{~h}$ of geraniol treatment (Fig. 2F). Therefore, our results indicate that autophagy is initiated before apoptosis in geraniol-treated PC-3 cells. Although Bcl- 2 exerts both antiapoptotic and antiautophagic function (28), our results show that geraniol induces autophagy before reduction of $\mathrm{Bcl}-2$ level, suggesting that $\mathrm{Bcl}-2$ is not a main factor of geraniolinduced autophagy. On the contrary, Bcl-2 level inversely well correlated with cleaved caspase- 3 and Bax.
Geraniol-induced apoptosis and autophagy cooperate to elicit cell death in PC-3 cells. Because apoptosis is not the sole cell death mechanism in geraniol-treated PC-3 cells (Fig. 1C), we assessed the significance of autophagy in geraniol-induced cell death. Treatment with zVAD or 3-MA (an autophagy blocker) alone partially rescued geraniol-induced cell death, whereas the combined treatment of zVAD and 3-MA restored cell growth $\leq 85 \%$ (Fig. 3A). When siRNA against ATG5 (siATG5) was used instead of 3-MA, we observed that the combined treatment of zVAD and siATG5 markedly restored cell growth compared to either treatment alone (Fig. 3B). These results indicate that apoptosis and autophagy cooperate to cause cell death in geraniol-treated cells.

We examined the inter-relationship between apoptosis and autophagy in geraniol-induced cell death. Inhibition of autophagy using 3-MA did not affect apoptosis, which was determined using caspase-3 activity assays (Fig. 4A and C). Inhibition of apoptosis using zVAD did not alter autophagy, which was measured using GFP-LC3 puncta formation assays (Fig. 4B and D). These results suggest that autophagy and apoptosis occur independently of each other in geraniol-treated PC-3 cells. However, these processes functioned as cooperative partners to induce cell death.

The effects of geraniol is unrelated to $H M G$-CoA reductase and TRPM8 pathways in PC-3 cells. Geraniol inhibits the activity of $\mathrm{HMG}-\mathrm{CoA}$ reductase, which catalyzes the production of mevalonate from HMG-CoA (6). Therefore, we tested whether mevalonate deficiency is attributable for geraniol-induced cell death. MTT assays showed that mevalonate supplementation to the culture medium did not rescue geraniol-induced cell death (Fig. 5).

Certain types of monoterpenes have agonistic activity for the TRPM8 calcium channel (7). To examine the involvement of TRPM8 in geraniol-induced cell death, we performed wholecell patch clamp analysis in TRPM8-transfected HEK 293 cells. Only menthol evoked TRPM8 current (Fig. 6A), whereas other monoterpenes did not act as TRPM8 agonists (Fig. 6B-D). Compared to other monoterpenes (Fig. 6E-G), geraniol had the most weakly elevated cytoplasmic calcium levels (Fig. 6H). 
A

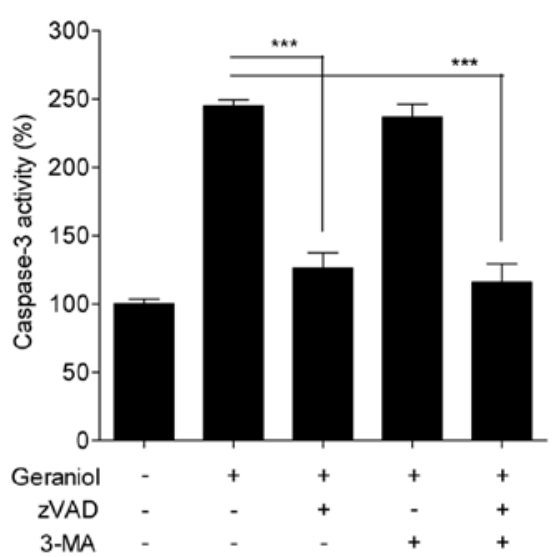

B

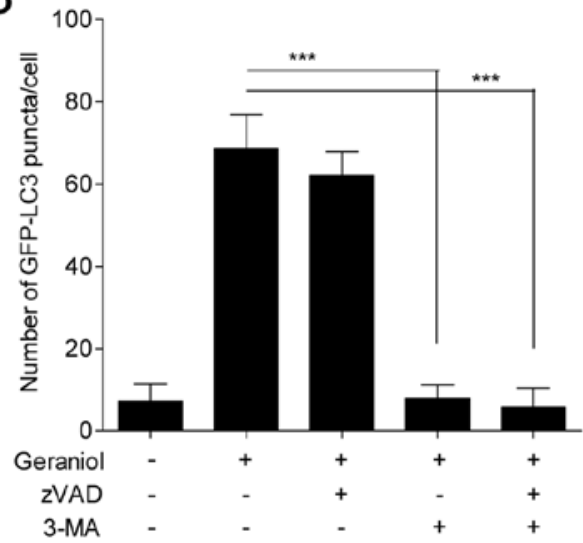

C

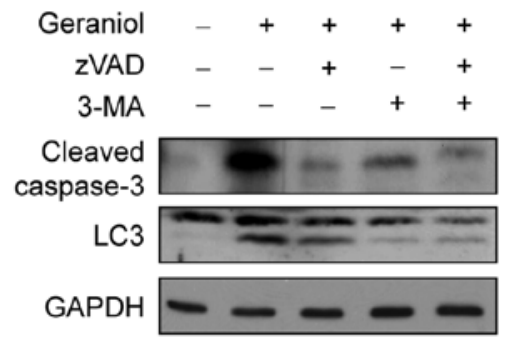

D

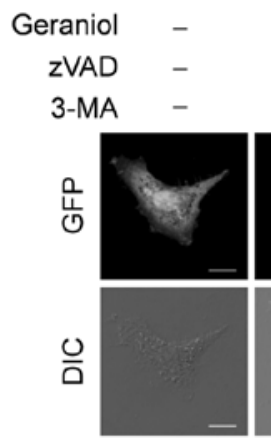

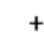

$-$

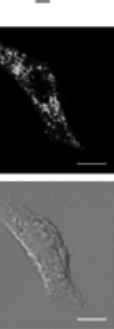

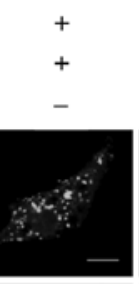
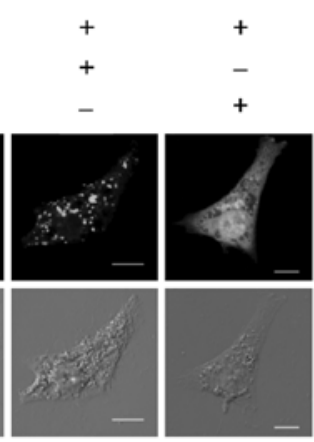

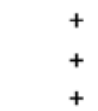

Figure 4. Apoptosis and autophagy occur independently of each other in geraniol-treated PC-3 cells. (A) The cells were incubated with geraniol (1 mM), zVAD $(10 \mu \mathrm{M})$, and/or 3-MA $(2 \mathrm{mM})$ for $48 \mathrm{~h}$ prior to caspase-3 activity assay. The data are expressed as the mean $\pm \mathrm{SEM}$ (n=4). (B) The cells were incubated with geraniol $(1 \mathrm{mM})$, zVAD $(10 \mu \mathrm{M})$, and/or 3-MA $(2 \mathrm{mM})$ for $20 \mathrm{~h}$ prior to quantifying the number of GFP-LC3 puncta. The data are expressed as the mean \pm SEM ( $=20)$. ${ }^{*} \mathrm{P}<0.05,{ }^{* * *} \mathrm{P}<0.005$. (C) Mevalonate supplementation does not rescue geraniol-induced cell death in PC-3 cells. The cells were treated with geraniol (1 mM) and/or mevalonate $(500 \mu \mathrm{M})$ for $72 \mathrm{~h}$ prior to MTT assays. Cell growth was expressed as a relative value compared to that of the untreated cells which was set to $100 \%$. The data were expressed as the mean $\pm \operatorname{SEM}(\mathrm{n}=4)$. (C and D) The cells were treated with geraniol (1 mM), zVAD (10 $\mu \mathrm{M})$, and/or 3-MA (2 mM) for 60 h (for apoptosis) or $20 \mathrm{~h}$ (for autophagy) prior to Western blot analyses (C) or confocal microscopic analyses (D).

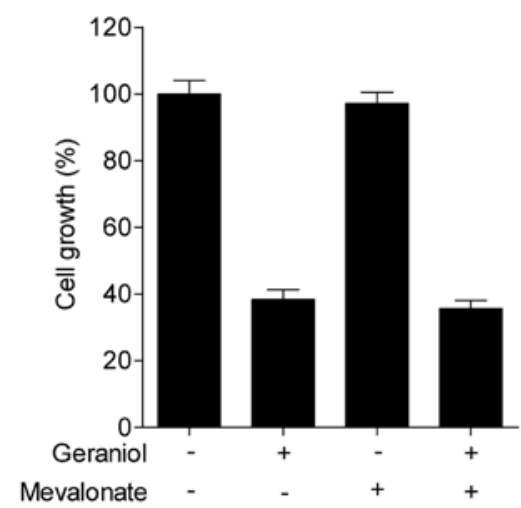

Figure 5. Mevalonate supplementation does not rescue geraniol-induced cell death in PC-3 cells. The cells were treated with geraniol $(1 \mathrm{mM})$ and/or mevalonate $(500 \mu \mathrm{M})$ for $72 \mathrm{~h}$ prior to MTT assays. Cell growth was expressed as a relative value compared to that of the untreated cells which was set to $100 \%$. The data are expressed as the mean $\pm \operatorname{SEM}(n=4)$.

Because linalool, cineol, or geraniol are not TRPM8 agonists, our results suggest that these monoterpenes elevate cytoplasmic calcium levels increased via TRPM8-independent mechanisms. Taken together, these results suggest that geraniol-induced cell death is not mediated by mevalonate deficiency and TRPM8 activation.

Geraniol inhibits AKT-mTOR signaling in PC-3 cells. Aberrant activation of AKT and mTOR following PTEN loss and/or androgen receptor inhibition has been observed in prostate cancer $(20,29)$ and mediates the inhibition of apoptosis and autophagy $(15,16)$. Therefore, we investigated whether geraniol inhibited AKT-mTOR signaling. As shown in Fig. 7A, geraniol inhibited AKT and its downstream effectors, including mTOR, $\mathrm{S} 6 \mathrm{~K}$, and 4EBP, in a concentration-dependent manner. AKT inhibition was observed following treatment with geraniol for $1 \mathrm{~h}$ and was sustained thereafter (Fig. 7B). However, geraniol did not activate MAP kinases, including ERK, JNK, and p38 kinase (Fig. 7C). In addition, geraniol specifically inhibited AKT/mTOR signaling compared to cineol (Fig. 7D).

We examined the mechanisms by which geraniol reduced AKT phosphorylation. We demonstrated that geraniol did not inhibit the phosphorylation of PDK1, which is an AKT upstream kinase (Fig. 7E). We investigated whether phosphatase pathways were involved in geraniol-induced AKT inhibition. Okadaic acid, which is a PP2A inhibitor, completely restored AKT phosphorylation compared to tautomycin, which is a PP1 inhibitor, in geraniol-treated cells (Fig. 7F). These results indicate that 

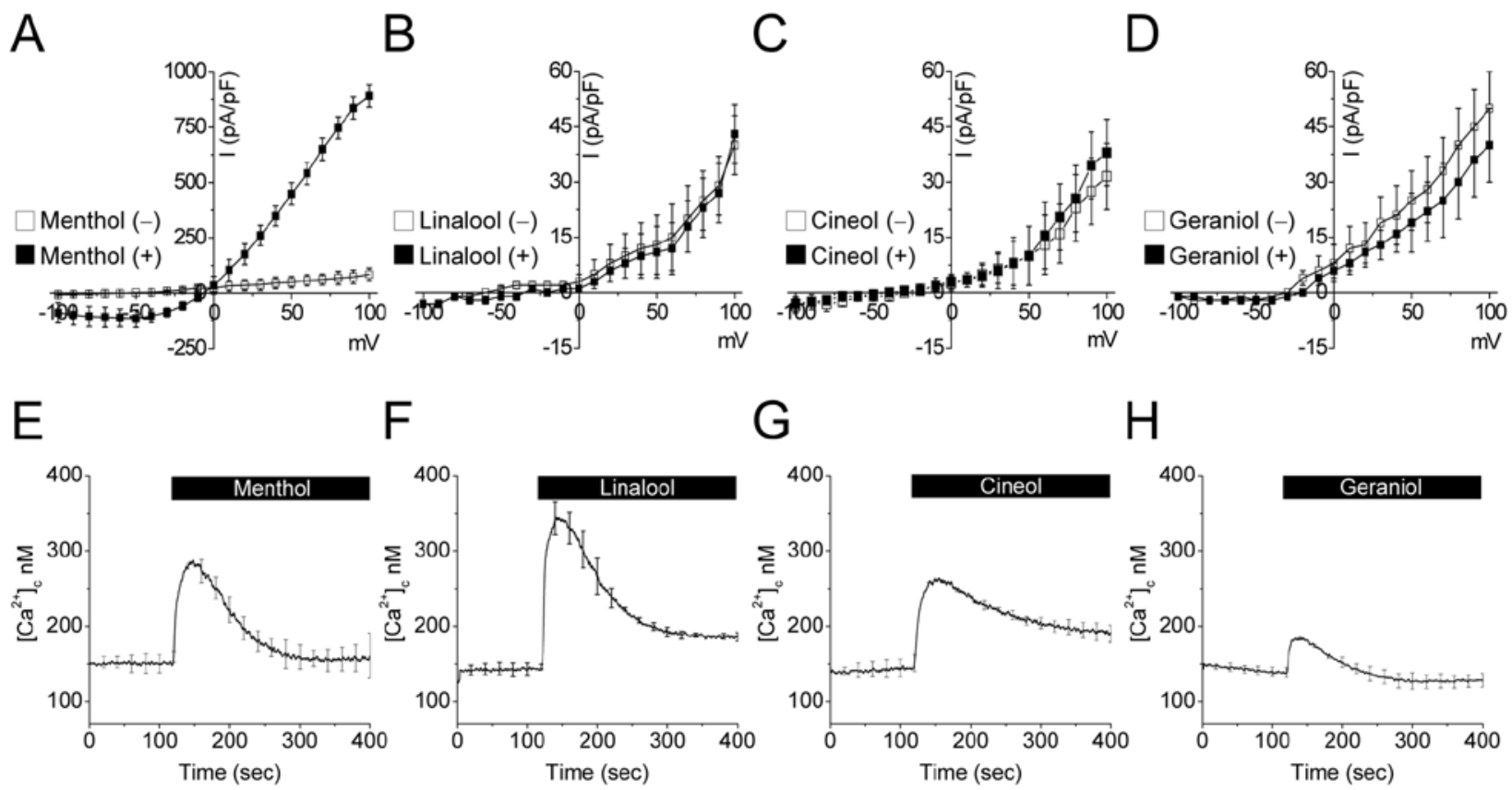

Figure 6. TRPM8 activation is not involved in geraniol-induced cell death in PC-3 cells. (A-D) The HEK 293 cells overexpressing human TRPM8 were used to evaluate change in the current-voltage relationship following treatment with the indicated monoterpenes $(1 \mathrm{mM}$ each). Note the different scales of the $\mathrm{y}$-axes. The data are expressed as the mean \pm SEM $(n=12)$. (E-H) The cytoplasmic calcium concentration was measured using Fura-2-AM dye in PC-3 cells that were treated with monoterpene (1 mM each). The data are expressed as the mean \pm SEM $(\mathrm{n}=12)$.

A

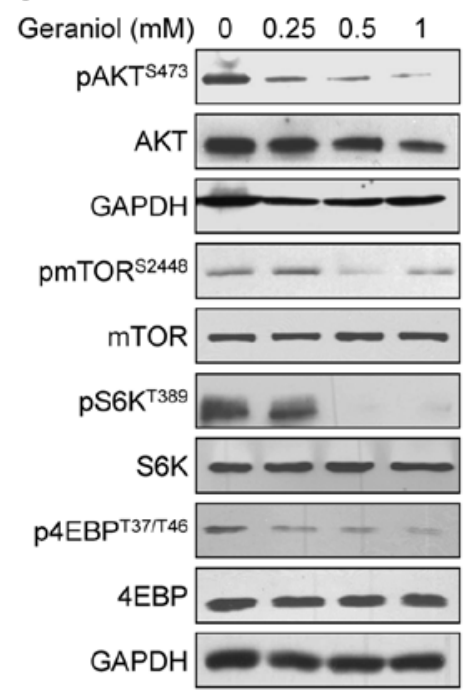

$\mathrm{E}$

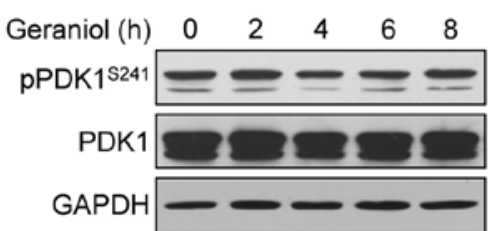

B

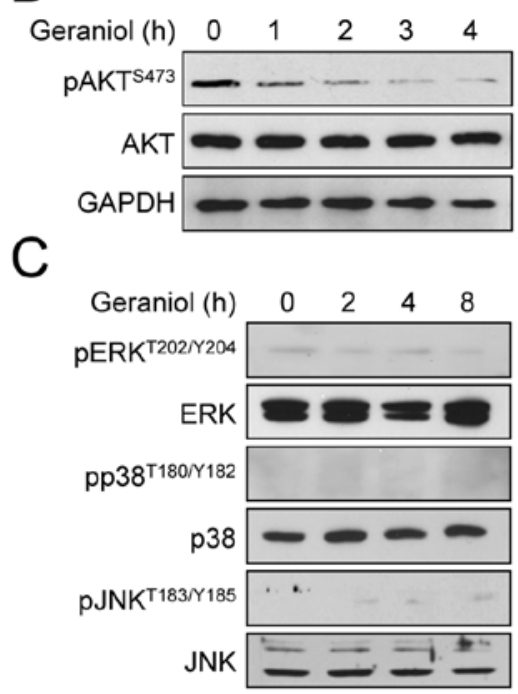

$\mathrm{F}$

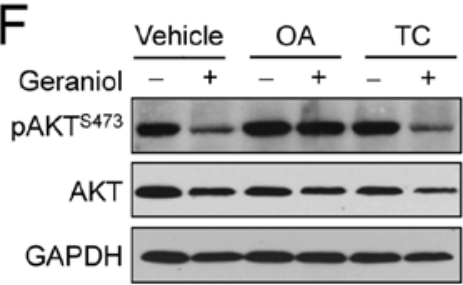

D

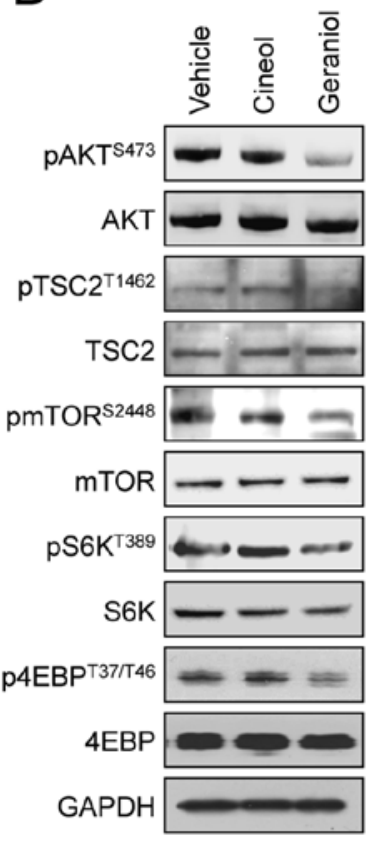

Figure 7. Geraniol inhibits the AKT-mTOR pathway in PC-3 cells. (A) The cells were treated with geraniol at the indicated concentrations for 4 h prior to Western blot analyses. (B-E) The cells were incubated with geraniol or cineol (1 mM) for the indicated times or $4 \mathrm{~h}$. (F) The cells were pretreated with okadaic acid (OA, $100 \mathrm{nM})$, or tautomycin $(\mathrm{TC}, 500 \mathrm{nM})$ for $30 \mathrm{~min}$ followed by treatment with geraniol $(1 \mathrm{mM})$ for $4 \mathrm{~h}$.

geraniol inhibits AKT signaling via regulation of the PP2A pathways.
Geraniol activates AMPK signaling in PC-3 cells. Because mTOR activity is also inhibited by AMPK signaling (22), we 


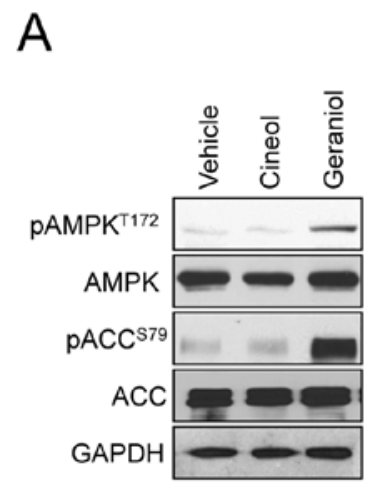

B

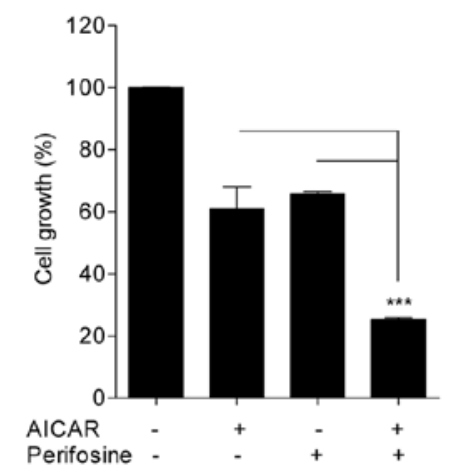

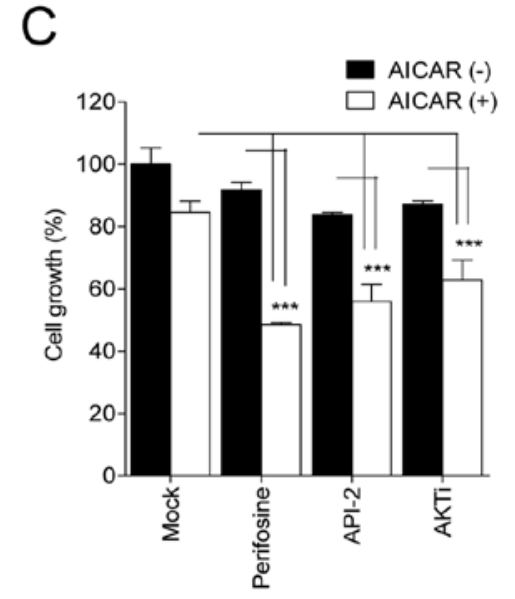

Figure 8. AMPK activation enhances the ability of AKT inhibitors to suppress tumor growth in PC-3 cells. (A) The cells were treated with geraniol at the indicated concentrations for $4 \mathrm{~h}$ prior to Western blot analyses. (B) The cells were treated with AICAR (1 mM) and/or perifosine $(5 \mu \mathrm{M})$ for $48 \mathrm{~h}$ prior to MTT assays. Cell growth was expressed as a relative value compared to that of the untreated cells which was set to $100 \%$. The data are expressed as the mean \pm SEM ( $\mathrm{n}=3$ ). ${ }^{* * * *} \mathrm{P}<0.005$. (C) The cells were treated with AICAR $(100 \mu \mathrm{M})$ and/or perifosine $(2 \mu \mathrm{M})$, AKTi-1/2 $(2 \mu \mathrm{M})$, or API-2 $(5 \mu \mathrm{M})$ for $48 \mathrm{~h}$ prior to MTT assays. The data are expressed as the mean \pm SEM $(n=4) .{ }^{* * * *} \mathrm{P}<0.005$.

examined whether geraniol activates AMPK. Compared to cineol, geraniol activated AMPK and increased the phosphorylation of ACC, an AMPK substrate (Fig. 8A). These results indicate that geraniol inhibits mTOR pathways by inhibiting AKT and activating AMPK signaling.

Either AKT inhibition or AMPK activation suppresses prostate cancer growth (23). Therefore, we investigated whether the combined modulation of AKT and AMPK is more effective than either alone in prostate cancer therapy. MTT assays showed that combined treatment of perifosine (an AKT inhibitor) and AICAR (an AMPK activator) markedly suppressed cell growth compared to either treatment alone (Fig. 8B). In addition, AICAR enhanced the anticancer activities of AKT inhibitors at sub-optimal doses (Fig. 8C). These results indicate that the concurrent modulation of AKT and AMPK activities may be a promising strategy for prostate cancer treatment.

\section{Discussion}

A strategy to restore the ability to induce cell death is needed to improve therapy for castration-resistant prostate cancer. However, cell death is a complex phenomenon that is independently or interactively regulated by multiple cellular and molecular mechanisms $(11,12)$. In this study, we described four main findings: i) geraniol induced apoptosis and autophagy, which occurred independently of each other; ii) combined inhibition of apoptosis and autophagy restored cell growth in geraniol-treated cells; iii) geraniol inhibited AKT and activated AMPK signaling; and iv) the combined modulation of AKT and AMPK is more effective than either alone in prostate cancer therapy. Therefore, our findings provide a basis for future investigations that are aimed at elucidating the role of apoptosis and autophagy in prostate cancer therapy.

We showed that combined inhibition of apoptosis and autophagy markedly restored cell growth, compared to inhibition of either process alone. These results suggest that these processes synergistically interact with each other to induce cell death. Our time-course analysis showed that autophagy preceded apoptosis in geraniol-treated cells. However, the results also suggest that apoptosis and autophagy are independently processes. Taken together, our results suggest that although these processes do not affect each other, the cooperation of apoptosis and autophagy after their induction is crucial to elicit cell death. Further studies using geraniol as a chemical probe may assist in understanding the phenotypic complexity of the relationship between apoptosis and autophagy at a molecular level.

Apoptosis and autophagy share common molecular regulators and pathways $(14,30)$. However, the phenotypic outcome of the interaction between these processes is extremely complex because apoptosis and autophagy exhibit synergistic or antagonistic effects on each other in cell death $(12,13)$. The mechanisms that generate this phenotypic complexity have not been well characterized. Furthermore, autophagy that is induced by clinically available anticancer agents has been reported to protect cancer cells from apoptotic cell death depending on the cell type and/or context $(31,32)$. Therefore, understanding the crosstalk between apoptosis and autophagy may be crucial for the development of therapeutic strategies to improve the efficacy of anticancer agents.

AKT inhibition and AMPK activation commonly resulted in mTOR inhibition (22). Our results showed that the combined effect of AKT inhibition and AMPK activation is more potent in suppressing prostate cancer growth than either alone. Because mTOR inhibition (e.g., rapamycin treatment) usually induces growth arrest rather than cell death $(33,34)$, the combined effects of AKT inhibition and AMPK activation seem to be mediated by various tumor suppressive signaling pathways in addition to $\mathrm{MTOR}$ inhibition. AKT or mTOR inhibition is not clinically successful in prostate cancer treatment (35). Therefore, our findings provide insight into future investigations that are aimed at developing the anticancer strategies co-targeting AKT and AMPK for prostate cancer therapy.

\section{Acknowledgements}

The human TRPM8 construct was kindly provided by Dr Barbara A. Niemeyer (University of Saarland). This research was supported by the National Research Foundation of Korea 
(NRF) funded by the Korea government (MEST) (2009-0086652 and 2010-0019472), and by a grant from the Seoul National University Hospital Research Fund (03-2009-019-0).

\section{References}

1. Lapczynski A, Bhatia SP, Foxenberg RJ, Letizia CS and Api AM: Fragrance material review on geraniol. Food Chem Toxicol 46 (Suppl. 11): S160-S170, 2008

2. Wiseman DA, Werner SR and Crowell PL: Cell cycle arrest by the isoprenoids perillyl alcohol, geraniol, and farnesol is mediated by p21(Cip1) and p27(Kip1) in human pancreatic adenocarcinoma cells. J Pharmacol Exp Ther 320: 1163-1170, 2007.

3. Carnesecchi S, Schneider Y, Ceraline J, et al: Geraniol, a component of plant essential oils, inhibits growth and polyamine biosynthesis in human colon cancer cells. J Pharmacol Exp Ther 298: 197-200, 2001.

4. Ong TP, Heidor R, De Conti A, Dagli ML and Moreno FS: Farnesol and geraniol chemopreventive activities during the initial phases of hepatocarcinogenesis involve similar actions on cell proliferation and DNA damage, but distinct actions on apoptosis, plasma cholesterol and HMGCoA reductase. Carcinogenesis 27: 1194-1203, 2006.

5. Kim SH, Bae HC, Park EJ, et al: Geraniol inhibits prostate cancer growth by targeting cell cycle and apoptosis pathways. Biochem Biophys Res Commun 407: 129-134, 2011.

6. Yu SG, Hildebrandt LA and Elson CE: Geraniol, an inhibitor of mevalonate biosynthesis, suppresses the growth of hepatomas and melanomas transplanted to rats and mice. J Nutr 125: 2763-2767, 1995.

7. Behrendt HJ, Germann T, Gillen C, Hatt H and Jostock R: Characterization of the mouse cold-menthol receptor TRPM8 and vanilloid receptor type-1 VR1 using a fluorometric imaging plate reader (FLIPR) assay. Br J Pharmacol 141: 737-745, 2004.

8. Jemal A, Bray F, Center MM, Ferlay J, Ward E and Forman D: Global cancer statistics. CA Cancer J Clin 61: 69-90, 2011.

9. Harris WP, Mostaghel EA, Nelson PS and Montgomery B: Androgen deprivation therapy: progress in understanding mechanisms of resistance and optimizing androgen depletion. Nat Clin Pract Urol 6: 76-85, 2009.

10. Chen CD, Welsbie DS, Tran C, et al: Molecular determinants of resistance to antiandrogen therapy. Nat Med 10: 33-39, 2004.

11. Lockshin RA and Zakeri Z: Apoptosis, autophagy, and more. Int J Biochem Cell Biol 36: 2405-2419, 2004.

12. Eisenberg-Lerner A, Bialik S, Simon HU and Kimchi A: Life and death partners: apoptosis, autophagy and the cross-talk between them. Cell Death Differ 16: 966-975, 2009.

13. Thorburn A: Apoptosis and autophagy: regulatory connections between two supposedly different processes. Apoptosis 13: 1-9, 2008.

14. Maiuri MC, Zalckvar E, Kimchi A and Kroemer G: Self-eating and self-killing: crosstalk between autophagy and apoptosis. Nat Rev Mol Cell Biol 8: 741-752, 2007.

15. Danial NN and Korsmeyer SJ: Cell death: critical control points. Cell 116: 205-219, 2004.

16. Wang S, Gao J, Lei Q, et al: Prostate-specific deletion of the murine Pten tumor suppressor gene leads to metastatic prostate cancer. Cancer Cell 4: 209-221, 2003.
17. Jiao J, Wang S, Qiao R, et al: Murine cell lines derived from Pten null prostate cancer show the critical role of PTEN in hormone refractory prostate cancer development. Cancer Res 67: 6083-6091, 2007.

18. Hollander MC, Blumenthal GM and Dennis PA: PTEN loss in the continuum of common cancers, rare syndromes and mouse models. Nat Rev Cancer 11: 289-301, 2011.

19. Shen MM and Abate-Shen C: Pten inactivation and the emergence of androgen-independent prostate cancer. Cancer Res 67: 6535$6538,2007$.

20. Taylor BS, Schultz N, Hieronymus H, et al: Integrative genomic profiling of human prostate cancer. Cancer Cell 18: 11-22, 2010.

21. Chen M, Pratt CP, Zeeman ME, et al: Identification of PHLPP1 as a tumor suppressor reveals the role of feedback activation in PTEN-mutant prostate cancer progression. Cancer Cell 20: 173-186, 2011.

22. Inoki K, Zhu T and Guan KL: TSC2 mediates cellular energy response to control cell growth and survival. Cell 115: 577-590, 2003.

23. Zhou J, Huang W, Tao R, et al: Inactivation of AMPK alters gene expression and promotes growth of prostate cancer cells. Oncogene 28: 1993-2002, 2009.

24. Kim SH, Nam JH, Park EJ, et al: Menthol regulates TRPM8independent processes in PC-3 prostate cancer cells. Biochim Biophys Acta 1792: 33-38, 2009.

25. Park EJ, Kim SH, Kim BJ, Kim SY, So I and Jeon JH: Menthol enhances an antiproliferative activity of 1alpha,25-dihydroxyvitamin $\mathrm{D}(3)$ in LNCaP cells. J Clin Biochem Nutr 44: 125-130, 2009.

26. Gozuacik D and Kimchi A: Autophagy as a cell death and tumor suppressor mechanism. Oncogene 23: 2891-2906, 2004.

27. Codogno P and Meijer AJ: Autophagy and signaling: their role in cell survival and cell death. Cell Death Differ 12 (Suppl. 2): 1509-1518, 2005.

28. Levine B, Sinha S and Kroemer G: Bcl-2 family members: dual regulators of apoptosis and autophagy. Autophagy 4: 600-606, 2008.

29. Mulholland DJ, Tran LM, Li Y, et al: Cell autonomous role of PTEN in regulating castration-resistant prostate cancer growth. Cancer Cell 19: 792-804, 2011.

30. Fimia GM and Piacentini M: Regulation of autophagy in mammals and its interplay with apoptosis. Cell Mol Life Sci 67: 1581-1588, 2010.

31. Dalby KN, Tekedereli I, Lopez-Berestein G and Ozpolat B: Targeting the prodeath and prosurvival functions of autophagy as novel therapeutic strategies in cancer. Autophagy 6: 322-329, 2010.

32. Chen S, Rehman SK, Zhang W, Wen A, Yao L and Zhang J: Autophagy is a therapeutic target in anticancer drug resistance. Biochim Biophys Acta 1806: 220-229, 2010.

33. Huang S and Houghton PJ: Targeting mTOR signaling for cancer therapy. Curr Opin Pharmacol 3: 371-377, 2003.

34. Hidalgo $M$ and Rowinsky EK: The rapamycin-sensitive signal transduction pathway as a target for cancer therapy. Oncogene 19: 6680-6686, 2000.

35. Engelman JA: Targeting PI3K signalling in cancer: opportunities, challenges and limitations. Nat Rev Cancer 9: 550-562, 2009. 